\title{
Pesticide Applications-Threat to Ecosystems
}

\author{
Surendra Kumar Yadav \\ School of Studies in Environment Management, Vikram University, Ujjain 456 010, \\ Madhya Pradesh, India \\ Cell: 91-9753791709; E-mail: sk_yadav11043@yahoo.com
}

KEYWORDS Pesticides. Ecosystems. Food Chain. Integrated Pest Management

\begin{abstract}
Many pesticides and chemicals are not biodegradable, and due to bioaccumulation, can enter into food chain and ultimately affect human and animal health. Environmental exposure of pesticides to humans through ecosystems may be during cropping/ agriculture practices, consumption of food materials or air inhalation etc. Without pesticides or agricultural chemicals (herbicides, insecticides, rodenticides, fungicides and plant growth regulators), crop yield could drop by as much as a third and food prices would increase by as much as $75 \%$. In India, 145 pesticides are registered for use at present and production has increased to approximately 85,000 metric tones. Though consumption in India is just about $0.5 \mathrm{~kg} / \mathrm{ha}$, but $51 \%$ food commodities are contaminated pesticide residues. Toxicity of pesticide is measured according to a number of indices (such as oral and dermal LD50) based on tests carried out on laboratory animals. They act mainly on receptors. In agriculture, mixing-loading step is the most contaminated task in open field accounting for two-thirds of the total daily exposure. Adverse health effects include acute and persistent injury to the nervous system, lung damage, injury to reproductive organs, and dysfunction of the immune and endocrine systems, birth defects and cancer. Even small amounts of some of these chemicals cause death; disrupt hormones and reduce the ability to successfully reproduce; and have been associated with specific cancers. Better understanding of the patterns of exposure, the underlying variability within the human population, and the links between the animal toxicology data and human health effects will improve the evaluation of the risks to human health posed by pesticides. Improving epidemiology studies and integrating this information with toxicology data will allow the human health risks of pesticide exposure to be more accurately judged by public health policy makers. There is need to educate all farmers for judicious use of pesticides, use of biotechnology and bio-pesticides, use of pesticides obtained from natural products such as Azadirachta indica (neem) via proper application of integrated pest management (IPM) programs would positively reflect on human health, agriculture, biodiversity and ecosystem management to minimize environmental problems caused by pesticides.
\end{abstract}

\section{INTRODUCTION}

Pesticide use has resulted in acute and chronic ecological damage either by direct injury such as birds and fish or by indirect effects such as elimination of natural enemies; particularly long-lasting effects have included the depression or stimulation of reproduction in organisms. Ecohealth approaches help translate research findings into policy and action. Ecohealth — short for "Ecosystem Approaches to Human Health" - is a research framework that addresses how human health and environmental quality are determined by complex relationships among different components of an ecosystem. It is used to explore how human health can be protected and improved through more sustainable ecosystem management. India suffers from double burden of pesticides - acute as well as chronic. Currently in India, the regulatory regime for pesticide is failing to ensure prudent tradeoff. Annually about $8,000-10,000$ cases and 1,000 plus fatality are in India. In Andhra Pradesh, the Government reported 200 odd cases from the entire state, whereas in Warangal alone, more than a thousand cases were reported in government hospitals alone and hundreds died due to poisoning during the same period. Pesticides/Pesticides Formulations banned in India are Aldrin, Benzene Hexachloride, Calcium Cyanide, Chlordane, Copper Acetoarsenite, CIbromochloropropane, Endrin, Ethyl Mercury Chloride, Ethyl Parathion, Heptachlor, Menazone, Nitrofen, Paraquat Dimethyl Sulphate, Pentachloro Nitrobenzene, Pentachlorophenol, Phenyl Mercury Acetate, Sodium Methane Arsonate, Tetradifon, Toxafen, Aldicarb, Chlorobenzilate, Dieldrine, Maleic Hydrazide, Ethylene Dibromide TCA (Trichloro acetic acid). Pesticide / Pesticide formulations banned for use but their manufacture is allowed for export for Nicotine Sulfate and Captafol 80\% Powder. Pesticide formulations banned for import, manufacture and use for Methomyl 24\% L, Methomyl 12.5\% L, Phosphamidon 85\% SL and Carbofuron 50\% SP. Pesticides withdrawn are Dalapon, Ferbam, Formothion, Nickel Chloride, Paradichlorobenzene (PDCB), Simazine, Warfarin and Metoxuron.

Assessing the toxic effects of pesticides on ecosystems is difficult, because so many species 
and processes are interacting. When significant changes in important ecological parameters become apparent only after a long time period, they are frequently undetectable in short-term experiments. Furthermore, observations made in one location may not apply to other sites because of variation among ecosystems. Finally, highly managed ecosystems may be more or less sensitive to a pesticide than a more natural community in the same site. Ecological consequences of pesticides can be studied at several levels viz., (a) accidental pesticide contamination of the environment may provide clues about how populations might be affected at the lower recommended use levels, (b) testing of sensitive species can indicate whether new biological or chemical pesticides will likely have side effects, (c) relying on experimentally created plots, field studies can be designed to measure the effects of pesticides on ecological parameters such as species diversity, energy flow, decomposition, tropic structure on indicator or monitored species, and, (d) laboratory experiments can be performed with individuals, populations, or model ecosystems constructed from combinations of species chosen to represent the numerous components of natural ecosystems.

Pest resurgence has been observed following applications of select pesticides that reduce natural enemy populations and subsequently increase pest populations. Field experiments have detected pesticidal effects on interspecies relationships such as predation. At the ecosystem level, the challenge is to determine whether pesticide use causes changes in viability. Soil biotas have been observed to be affected in a complex manner; that is, some species increase in numbers, while others are reduced by injury. Field methods that exist for such ecological studies have been adapted to identify effects of pesticides, and have been applied most often to the soil biota portion of the ecosystem. Thus, long-term observation is required for such studies (Wilson 1996). Similar effects take place above ground and in a plant host. To understand the effects of pesticides on ecosystems, it is necessary to examine a spectrum of effects from lethality to subtle changes on reproduction, behaviour, and vital organ physiology (Wilson 1999). Responses may vary between closely related species or even 'biotypes' of a species, so that careful identification of test specimens is critical to learning much in depth about the genetic characteristics of the species of interest. Pesticides affect ecosystems by disrupting natural equilibrium; their effects can be observed by measuring the stability of populations, nutrient cycling, species diversity, interspecies food chains, primary production in and energy flow through tropic levels, and pollination. Some pesticides exert their effects on particular components of an ecosystem (Yadav 2007); for instance, some herbicides affect primary production in plants, and persistent organochlorine insecticides (such as DDT) bioaccumulate in higher tropic levels such as predators. Broadspectrum OP and carbamate insecticides with high acute toxicity to many species may acutely alter energy flow as well as other ecological parameters.

Environmental and toxicological studies suggest that agricultural practices are an important route by which eagles may be poisoned by pesticides. In a study, more than 100 eagles have been observed at the landfill but no poisoning incidents have been directly linked with feeding there (J Elliott, CWS, Unpublished data, August 1999). During the winter of 1995-96, an estimated 20,000 Swainson's Hawks died in Argentina after feeding on contaminated grasshoppers. This represented approximately 5\% of the global Swainson's Hawk population, demonstrating the importance of regulating and monitoring pesticide usage (Henny et al. 1998). Eagles are highly visible top predators whose injuries and deaths are more likely to be noticed by the public.

Risk assessment is comprised of 4 consecutive steps, i.e. hazard identification, hazard characterization, exposure assessment and risk characterization (WHO 1995). All standards vary between countries, the situation encourages the export of hazardous materials, and hazardous industries, from the developed to developing countries (Jeyaratnam 2002). Residues of organocholorine insecticides, like DDT has been detected in human and his environment the world over (Bhatnagar 2001). Farmers must be educated for judicious use of pesticides, and other alternatives such as use of biotechnology, use of biopesticides, and use of pesticides obtained from natural products such as neem should be encouraged (Gupta 2004). Adverse health effects of pesticides exposures includes acute and persistent injury to nervous system, lung damage, injury to the reproductive organs, dysfunction 
of immune and endocrine systems, birth defects, and cancer (Mansour 2004). Interactions between different types of pesticides have received considerable alteration (Dikshith and Raizada 1994; Krishnan and Brodeur 1994; Cantalamessa 1993; Mansour and Heikal 2001). Role of toxicity and exposure assessment in mechanisms of susceptibility for children's health risk is also important priority (Faustman et al. 2000).

Ecosystems are integrated and stable systems; they include humans, all other species on this planet, and basic biotic and abiotic processes. Prediction and assessment of ecological impacts caused by pesticides alert humans to the dangers of some alterations to the environment. Components of ecosystems cannot be changed or destroyed without directly or indirectly impacting the human condition. It is to humans' benefit to consider all uses and risks of pesticides to ensure preservation of critical systems in the environment.

\section{MAGNITUDE DETERMINES IMPACT OF PESTICIDE}

There are about 1000 pesticide formulations in use throughout the world today; the listing of all hazards to ecosystems would be enormous, particularly with most individual pesticides having different effects on various species. Agriculture and forestry are the primary source of pesticides in ecosystems. Clearly delineating these effects is complicated by the fact that there are 5-10 million species in the environment. The United Nations offers a list of pesticides considered most hazardous to humans. While valuable, this list is limited, since it is restricted to acutely lethal doses in humans (based on studies in the rat and other laboratory species). In general, insecticides generally are the most toxic pesticides to the environment, followed by fungicides and herbicides. Exceptions exist for certain herbicides which are highly toxic, and are far more hazardous to the environment than are insecticides. The most hazardous pesticides include those that can be distinguished on the basis of either water or fat solubility. Water soluble compounds are easily transported out of the target area into ground water and streams; fat soluble chemicals are readily absorbed in insects, fish, and other animals, often resulting in extended persistence in food chains (Yadav 2007). Some of the most troublesome pesticides to the ecology are (a) insecticides: DDT, dieldrin, diazinon, parathion, and aldicarb; (b) herbicides: 2-4-D, atrazine, paraquat, and glyphosate, and (c) fungicides: benomyl, captan, mercury, copper, and pentachlorophenol.

About 5 million ton of pesticides are applied annually in the world, of which about 70 per cent is used for agriculture and the remainder by public health agencies and government agencies for vector control and by home owners. In many countries, agriculture and forestry occupy approximately 50 per cent of the land area. When croplands are treated, some impacts of pesticides occur on non-target terrestrial and aquatic ecosystems, as well as on adjoining agro ecosystems. Forests are important wildlife habitats. Two broad classes of pesticides are used in forests: insecticides to control insect pests, and herbicides used to suppress the growth of shrubs during the regeneration process. Over the last thirty-five years, two large-scale pest control programmes have been used in forests of eastern Canada and of the north-eastern United States in attempts to control spruce budworm; from 1980 to 1983 in Czechoslovakia, similar programmes were initiated to control the larch bud moth. In the latter programme, a mixture of pyriphosmethyl and permethrin eliminated many invertebrate species, but recovery was relatively rapid (Tonner et al. 1983). In eastern Canadian forests, DDT caused fish mortality (Kerswill and Edwards 1967) as a result of bioaccumulation through the food chain (Wilson 1995).

Lethal and indirect effects of decreasing the biomass of insect food would be expected to affect insectivores, but that has rarely been demonstrated. Extensive mortality of canopy-dwelling song birds has been observed with applications of phosphamidon and to a lesser extent, with fenitrothion; ground-nesting birds and small mammals were unaffected (Pearce et al. 1976). Generally, the safety margin of OPs for canopydwelling birds is small.

\section{Technologies in Pesticide Application}

Generally, less than 0.1 per cent of all pesticides applied reach the target pests, according to some estimates. If so, then a large fraction of applied pesticides may be available to contaminate water, soil, and atmosphere and may disrupt non-target species. One reason for the small amounts of pesticides that actually reach 
target pests is the requirement that plant surfaces be thoroughly covered with pesticides to control small arthropods (e.g., aphids and mites) and plant pathogens (fungi and bacteria). The manner in which pesticides are applied is influential in determining the nature and magnitude of injury that may occur in ecological species. Likewise, this process also greatly impacts the effectiveness of pesticides. To achieve this, thorough coverage of plant surfaces requires that sprayed particles be extremely small, a situation that favours dissemination by gentle winds for distances of several thousand meters. Achieving coverage of crop plant surfaces with pesticides is part of the problem, as is placing the pesticides into the target area.

Under ideal conditions using aircraft, for instance, less than 50 per cent of the pesticide applied by aircraft reaches the target crops. With ultra-low-volume sprays (ULV) by aircraft, drift is even more extensive, placing less pesticide in the target area. Under ideal weather conditions, less than 25 per cent of the ULV-applied pesticide reaches the target. Likewise, most drifts often contaminate untreated terrestrial and aquatic ecosystems. Large quantities of pesticides are applied to orchards, vineyards, and similar crops by employing air blast sprayers. Only about 65 per cent of the pesticide applied by use of this equipment reaches the target area, whereas 35 per cent goes elsewhere. Ground application equipment under normal weather conditions often place 7080 per cent of the sprayed pesticide into the target area. If, at the same time, a 'plastic blanket' were used to protect the crop and spray boom, perhaps 90 per cent or more of the pesticide could be placed in the target area.

The physical form of pesticide formulations (i.e., wick, dust, granule, and chemigration technologies), greatly influences the extent to which a pesticide will be placed on the target site and be distributed elsewhere. Dusts are highly susceptible to drift because of their extremely low weight. Seldom does 50 per cent of the applied dust remain in the target area when using ground equipment, even under ideal wind conditions. Granules have become a popular means of applying dry insecticide materials to the surface of croplands and pasture lands. However, granules pose major problems to non-target pests, particularly birds. Birds mistake the granules for gravel, and may consume them with disastrous results. Some pesticides can also be effectively applied in irrigation water; this practice is referred to as 'chemigration'. Although this is an effective, easy method of applying pesticides to agricultural crops, it is also a means of spreading pesticides in the environment and of poisoning non-target species. In particular, it can contaminate the drinking water of birds, mammals, invertebrates, and humans. Ground and surface waters are also readily contaminated by chemigration. Clearly, the need exists to improve pesticide application technology to assure that more pesticide reaches the target points and to reduce hazards to human and ecosystem.

\section{Risk for Many Species}

Risk has been demonstrated by many researchers. The susceptibility, such as crustaceans, is due to the similarity of their physiological sites of action among target insects and certain nontarget invertebrates; for example, AChE enzymes, octopamine receptors, and moulting processes are all similar insecticide targets in both pest and invertebrate. Terbufos, chlorpyrifos, and carbofuran are important insecticides for controlling corn rootworm larvae in soil; however, both terbufos and carbofuran are highly toxic to earthworms. Foliar applications of broad spectrum insecticides produce nearly total depletion of arthropod populations in crops such as cotton. While selectivity for herbivorous pests can be gained through systemic insecticides, some of these insecticides, such as aldicarb and oxamyl, are extremely toxic; thus, secondary poisoning can result in predators of treated pests. Some selectivity of insecticides can be achieved. For example, the insect development-inhibitor methoprene controls mosquito larvae at doses not toxic to most NTO aquatic invertebrate species. Substrains of Bacillus thuringiensis are selective for certain insects in a given taxonomic order: B.t. israeleasis for mosquito larvae and $B$. t. kurstaki for lepidopterous larvae. Some herbicides and fungicides also affect NTO invertebrates, although they are the exceptions. Bipyridilium herbicides have a non-selective cytotoxic action, as do dinitrophenol pesticides which uncouple oxidative phosphorylation.

For pesticide registration, data are required about toxicity to representative invertebrates such as Daphnia magna, earthworms, and decapod crutaceans. Test protocols are specified by various national and international organizations 
such as the US EPA, OECD, and FAO. Microorganisms are most susceptible to fungicides and bactericides aimed at target plant pathogens in the field. Some herbicides are effective against both fungi and bacteria by producing the same molecular lesions in each. Generally, microorganisms lack the physiological and molecular targets (e.g., photosynthetic pathways) that render them susceptible to insecticides and herbicides. Insecticides and herbicides can deplete the insect and plant hosts of microorganisms. Microorganisms are very important in the environmental detoxification and decomposition of pesticides. Soil ecosystems can change because of the enhanced capacity of select soils to degrade pesticides, as observed in the loss of effectiveness developed in certain soils after 10 to 20 years of continuous pesticide use.

\section{Pesticide Effect at Various Levels in Plants}

Pesticides impacts depend on level in the ecosystem. Ecosystems, whether plant or animal, can suffer from accrued damage to individuals. As an illustration, the complexities of such interactions among plants are described. Plants are exposed to pesticides, whether as target organisms such as pathogenic fungi. The routes of contact include the uptake from soil and water and deposition via atmospheric drift. Toxic and mutagenic effects and changes in the metabolism of plants (including formation of metabolites and residues capable of producing adverse effects) can be observed in plants, even with quantitative and qualitative differences resulting from variations in plant sensitivity, and with the numerous metabolic pathways of pesticide degradation. Differential sensitivity of plant species to toxic and genotoxic effects of pesticides has been shown to cause overall changes in species proportion among weeds in croplands and in natural plant communities, because of the reduced abundance of susceptible species and concurrent increases in naturally tolerant species. However, neither the eradication of a susceptible species nor the occurrence of a new plant species has been established in the experimental plots even after long-term (36 years) spraying with formulations such as the herbicide 2,4-D. The emergence among susceptible plant species of forms resistant to herbicides has become increasingly important. Pesticides can play a dual role as agents favouring the selection of pre- existing resistant mutants and as potential inducers of genetic changes. Genotoxic pesticides are potentially able to increase the pool of mutations in various qualitative and quantitative traits that could cause genetic instabilities of natural plant populations and in crop varieties.

Elements related to possible ecological damage include the formation of stable mutagenic and toxic metabolites and the accumulation of residues in plants that can be harmful to both human and animal populations via exposure through the food chain. This problem needs to be addressed despite the fact that in plants bound residues formed and incorporated into lignin, hemicellulose, and other carbohydrate components of the cell wall are usually less hazardous to the biosphere. Pesticides have produced changes in both plant metabolism and nutritional patterns which may have further detrimental effects on the ecology. Several mechanisms are known to lead to this consequence viz.: (1) Some herbicides, when applied at recommended dosages, have increased the attacks of insect pests and plant pathogens on crops. For example, when corn-growing areas were treated with 2,4-D at the recommended dosage of $1 \mathrm{~kg} / \mathrm{ha}$, the numbers of corn leaf aphids increased three-fold; corn borers were 26 per cent more abundant, and were 33 per cent larger than those insects present on untreated corn. Larger corn borers produce one-third more eggs, and thus contribute to the build-up of corn borers on corn. (2) The insecticides monocrotophos and phosphamidon increased concentrations of nitrogen and phosphorus in rice plants, and these changes were thought to contribute to resurgence in the numbers of the rice blue leafhopper. (3) Herbicides may increase damage due to plant pathogens. For instance, when corn was treated with a recommended dosage of 2,4-D (1 kg/ha), black corn smut grew five-fold larger than on untreated corn. Also, corn that was resistant to southern corn leaf blight lost its resistance to the blight when treated with 2,4-D. (4) Most nutrients, especially C, K, N, P, and S, are taken up by plants that, in turn, may be eaten by animals. These nutrients are eventually returned to the soil or atmosphere via decomposition of dead organisms. The amounts and forms of nutrients in soils and plants may be changed by pesticides, thereby altering the dynamics of these animals in the ecosystem. (5) Pesticides can alter the chemical make-up of plants. The changes that occur appear 
to be specific for both the plants and pesticides involved. For example, certain organochlorine insecticides have increased the amounts of some macro and micro-elements (Al, B, Ca, Cu, Fe, K, $\mathrm{Mg}, \mathrm{Mn}, \mathrm{N}, \mathrm{P}, \mathrm{Sr}$, and $\mathrm{Zn}$ ) of corn and beans. DDT, aldrin, endrin, and lindane have stimulated synthesis of the essential amino acids arginine, histidine, leucine, lysine, proline, and tyrosine in corn, but have decreased the content of tryptophan. The herbicide simazine increased water and nitrate uptake in barley, rye, and oat seedlings, resulting in increased plant weight and total protein content.

The toxic effects of pesticides have been measured in plants growing in separated experimental plots using endpoints such as frequency of species and individuals in each species, survival of plants, fresh and dry weight of plants at the harvest, and seed setting. Thus, the environmental problem arising from the use of herbicides may involve more than just the herbicide itself. Increasing insect pest and plant pathogen attacks on crops by using some herbicides may, in turn, lead to an increase in spraying of additional pesticides, like insecticides and fungicides. Methods are available to detect both non-genotoxicity and genotoxicity of pesticides in plants, including their effects on plant reproduction. Cytogenetic analyses of mitotic cells in roots and shoots, meiotic cells in pollen mother cells, and post meiotic cells in pollen represent the most frequently used methods to detect genetic changes caused in natural plant populations and communities or in plants growing in the field. Special plant assay systems are also available to monitor genotoxicity in situ. About 150160 pesticides have been tested on plant genotoxicity assay systems. About 90 per cent of the test agents produced some kinds of changes in the chromosome structure, about 60 per cent caused disturbances in the meiosis and seed set reduction, and about 70 per cent caused gene mutations. Compared with other assay systems (e.g., microbial cells, mammalian cell cultures, insects, and whole animals), plant assays appear to be the most efficient to detect pesticiderelated genotoxicity. Seasonal variations in cytogenetic endpoints have been correlated with the application of herbicide mixtures.

\section{Pesticide Effect at Various Levels in Animals}

The more obvious effects of pesticides (i.e., reproductive failure caused by DDT and mortality caused by carbamates) have, so far, been the basis of concern and regulatory action. The major adverse effects of organochlorine (OC) pesticides have been manifested through effects on reproduction. DDT has caused eggshell thinning in several high tropic level avian species and sufficient impact on reproduction to result in population declines (Risebrough 1986). Likewise, the effects on fish occurred largely during the reproductive cycle (i.e., at the time that the yolk sac was absorbed) (Burdick et al . 1967). By contrast, the major effect of OPs and carbamates has been direct mortality. The mechanism of action of these insecticides is the inhibition of AChE activity which causes the disruption of nerve function. Acute inhibition of 80 per cent and chronic inhibition of 50 per cent has been associated with mortality (Ludke et al. 1975; Hill and Fleming 1982). Other esterases are also inhibited, and the inhibition of brain neurotoxic esterase (NTE) has been related to delayed neuropathy (Johnson 1975). Lotti and Johnson (1978) compared the toxicity of inhibition of AChE and NTE for a range of OPs. The ratio of inhibition of the two enzymes varies over several orders of magnitude, but the degree of inhibition of AChE correlates well with the $\mathrm{LD}_{50}$ and that of NTE with delayed neurotoxic damage. For those pesticides causing neurotoxic effects, inhibition of NTE can be one to two orders of magnitude more sensitive than that of AChE. The relationship between dosage of the pesticide and degree of inhibition of AChE has been used to assess the impact of insecticide spray programme on forest songbirds (Mineau and Peakall 1987). The data show a severe collection bias in favour of birds with inhibition of the enzyme of less than 30 per cent. This manifestation is probably due to behavioural effects on birds having AChE inhibition greater than this value.

Because behaviour is the result of integration of many inputs, it has long been considered as a potentially sensitive indicator of pesticide toxicity (Warner et al. 1966). For OPs, behavioural alterations are demonstrated only at AChE inhibition of 4050 per cent (Grue et al. 1982; Rudolph et al. 1984). The behavioural effects, are not particularly sensitive, has been confirmed by a review of the effects of toxic chemicals on birds (Peakall 1985). While it can be stated that behavioural changes will reduce an organism's ability to survive in the wild, little direct evidence exists to confirm this hypothesis. In some cases, predation experiments 
have been conducted in the laboratory (Brown et al. 1985); there remains a major extrapolation to field conditions. Furthermore, in this experiment a significant increase in predation was not seen until the concentration equivalent to half the $\mathrm{LD}_{50}$ was used.

\section{Impacts on Functional Ecosystems}

Terrestrial and aquatic ecosystems are generally self-sufficient basic living units of nature that include a complex of species dependent on one another, and interacting with the physical-chemical environment. Pesticides may alter the structure (species richness, density, and biological diversity) and functional activities of an ecosystem. Some pesticides are capable of destroying some species totally or of significantly reducing the populations of others. When the diversity of the ecosystem is reduced sufficiently, then food chains may be shortened or altered in diverse ways. When food chains are changed, the stability of ecosystems may be reduced, leading a susceptible ecosystem to extinction by a variety of mechanisms such as invasion by other complexes of species. Thus, pesticides may alter the self-sufficient nature of natural ecosystems that include plants (producers), herbivores, parasites and predators, and decomposers. Energy fixed by the plants eventually becomes available to herbivores and other species that make up the ecosystem. A critical component of all ecosystems is energy flow. Plants collect energy from the sun for growth, metabolism, and reproduction. The more energy the plants collect, the more productive, diverse, and complex is the ecosystem. In aquatic ecosystems, eutrophicationa form of energy cyclingmay enhance or limit diversity. If a pesticide influences the growth of the plant populations, then the food/energy supply for the ecosystem is reduced, lowering its productivity and stability.

Another essential component of an ecosystem is the decomposition of organic matter. The basic elements (e.g., C, Ca, H, K, Mg, Mn, N, O, P) are vital to the proper functioning of all life, including ecosystems. Thus, the decomposers are essential to keep the vital nutrients in circulation for use and reuse by an ecosystem. Some essential nutrients are in the atmosphere. Plants and other organisms may obtain some essential elements $(\mathrm{C}, \mathrm{H}, \mathrm{O}, \mathrm{N})$ from the atmosphere for use throughout an ecosystem. Other nutrients may be obtained directly from soil or water, and are cycled through the biota. Pesticides may be capable of reducing the variability of one or more organisms involved in the recycling process in an ecosystem. If this occurs to a large extent in an ecosystem, it may function at such a reduced rate as to threaten the entire web in the ecosystem. Decomposers are organisms (microbes, earthworms, insects, etc.) responsible for the decomposition of organic compounds other than their nutrients. Methods to quantify ecological effects of pesticides on terrestrial and aquatic eco-systems are generally complex and costly, the magnitude and complexity being dependent on the nature of the pesticides and on the characteristics of the particular terrestrial and aquatic ecosystem in which pesticide is used.

The effects on ecosystem components seem most urgently to require assessment prior to pesticide registration, these are the effects on the tropic structure (plants, herbivores, parasites, and predators), and on decomposer organisms, because of the unique vulnerability demonstrated by these components for ecosystem integrity and survival. Any new pesticide, whether biological or chemical, should first be assessed for ecosystem effects in the particular agro-ecosystem of proposed primary use (e.g., an apple orchard or a cotton field). This approach requires the measurement of population dynamics for a representative number of resident species in addition to the target pests and their natural enemies. The number of species to be sampled should be in proportion to the complexity of the agro ecosystem of interest. Effects on decomposers can be determined by cellulosic sampling by litterbag methods, for a minimum of three growing seasons.

\section{DISCUSSION}

Farmers from developing countries are challenged by these changes, affecting livelihoods, food security, environment, and health. Irrigation, chemicals, and crop technology are transforming agriculture around the world. Using eco-health approaches, researchers can better understand the complex dynamics affecting health in agroecosystems and design more equitable and sustainable solutions. Pest resistance leads to environmental impacts through (1) total impact of a pesticide depends on toxicity, dosage and location (2) pests develop resistance, may have far reaching effects, and desirable insects also 
impacted (3) resurgences (pest population recovers and explodes) (4) secondary pest outbreaks (nonpests become pests as loose natural enemies, gain resistance to pesticides) (5) pesticide treadmill (chemicals don't eradicate pests, but lead to increased resistance and secondary outbreaks) (6) bioaccumulation: minimal exposure leads to increasing toxic levels over time, as chemicals collect in lipids (7) biomagnifications: multiplying effect of bioaccumulation through the food chain. Integrated Pest Management (IPM) goal is to minimize use of synthetic organic pesticides, without hurting crops by accounting for interacting sociological, economic and ecological factors; basically impact is reduced rather than eradication of pests. IPM methods (cultural and biological) are to give emphasis on extension programs to find best times (field monitoring), loss insurance to avoid insurance spraying, keeping in view of public policy, Laws and Provisions. Various National and International organizations/ Bodies [EPA, FDA and USDA, UN Food and Agriculture Organization (FAO), UNEP etc.] are working for formulations and amendments of related Acts and for issues for utilization of the pesticides. Pesticide types are basically (1) first generation pesticides: toxic metals (2) second generation pesticides: synthetic organic pesticides e.g. chlorinated hydrocarbons such as persistent DDT (3) nonpersistent pesticides (e.g. malathion, aldicarb). Impact of pesticides on ecosystems is assessed in terms of economic impact to food, health, structures; public desire for cosmetic products and types of pest control (herbicide, pesticide, rodenticide and fungicide). Approaches for pest management include chemical treatment, ecological control and integrated pest management (IPM).

Unintended spatial spread of genetic information is one of the major problems in modern agriculture. The vertical distribution of transgenic properties and the spatial spread of resistant weeds and pests are likely to develop under longterm pesticide use. These are complex systems that require an integrated view of population dynamics, genetics, and physical transport processes. Mathematical models may be utilized to support of risk assessment and to derive appropriate risk management strategies. In this contribution, we propose a generic modeling framework that provides an explicit simulation of the spatial patterns of gene flow through agroecosystems. Pollen dispersal is modeled with traditional transport equations from atmospheric physics that are applied to out crossing studies of genetically modified maize. Transport equations were coupled to models of population dynamics and genetics with partial differential equations that combine dispersal, growth and genetics. The overall model consists of a set of coupled partial differential equations for pollen dispersal, and the spatial and temporal dynamics of each biotype involved. Initial boundary value problems are set up for the dispersal of resistance in dependence on spatial spread patterns, which are solved by finite element methods

\section{CONCLUSION}

Approaches for pest management include chemical treatment, ecological control and integrated pest management (IPM). Non-chemical pest controls, such as biological, cultural, and environmental controls and host plant resistance, should be improved and used in order to reduce pesticide use. Alternative pest control methods include cultural control: non-chemical alteration of environmental factors e.g. hygiene, crop rotation; control by natural enemies: e.g. Brazilian weevils for water hyacinth (first step is conservation, then finding specific not general enemy); genetic control: breed resistance crops using chemical (e.g. Hessian fly on wheat) or physical barriers (e.g. hooked hairs), sterile males (e.g. tsetse fly), biotechnology and genetic engineering for transgenic crops (e.g. resistance to pest, or resistance to broad-spectrum herbicide); natural chemical control (isolate, ID, synthesize then use insects own hormones or pheromones to disrupt its life cycle. Impacts may be non-toxic and specific. Sex pheromones can be used to lead insects into traps, or confuse them. Further research is needed for understanding complexity of pesticides in ecosystems.

\section{REFERENCES}

Bhatnagar VK 2001. Pesticide pollution: Trends and perspectives. ICMR Bulletin, 31(9): 87-88.

Brown JA, Johansen RH, Colgan PW, Mathers RA 1985. Changes in the predator-avoidance behaviour of juvenile guppies (Poecilia reticulata) exposed to pentachlorophenol. Can J Zool, 63: 2001-2005.

Burdick GE Harris EJ, Dean HJ, Walker TM, Shea J, Colby D 1967. The accumulation of DDT in Lake Trout and the effect on reproduction. Trans Amer Fish Soc, 93: 127-136.

Cantalamessa F 1993. Acute toxicity of two pyrethroids, permethrine, and cypermethrin in neonatal and adult rats. Arch Toxicol, 67(7): 510-513. 
Dikshith TSS, Raizada RB 1994. Response of carbon tetrachloride pretreated rats to endosulfan, carbaryl and phosphamidon. Ind Health, 21(4): 263-272.

Elliott JE, Wilson LK, Langelier KL, Mineau P, Sinclair P 1999. Secondary poisoning of Birds of Prey by the Organophosphorus Insecticide, Phorate. Ecotoxicology 5:1-13.

Faustman EM, Silbernagel SM, Fenske RA, burbacher TM, Ponce RA 2000. Mechanism underlying children's susceptibility to environmental toxicants. Environ Health Perspect, 108 (Suppl. 1): 12-21.

Grue CE, Powell GVN, McChesney MJ 1982. Care of nestlings by wild female Starlings exposed to an organosphosphate pesticide. J Appl Eco, 19: 327-355.

Gupta PK 2004. Pesticide exposure- Indian scene. Toxicology, 198: 83-90.

Henny CJ, Mineau P, Elliott JE, Woodbridge B 1998. Raptor poisonings and current insecticide use: what do isolated kill reports mean to populations? In: N Adams, R Slotow (Eds.): Proc. 22 Int Ornithol Congr, Durban, University of Natal.

Hill EF, Fleming WJ 1982. Anticholinesterase poisoning of birds: Field monitoring and diagnostic of acute poisoning. Environ Toxicol Chem, 1: 27-38.

Jeyaratnam J 2002. Development of methods and models, and good practices. People Work Rep, 49: 87-91.

Johnson MK 1975. The delayed neuropathy caused by some organophosphate esters: Mechanism and challenge. Crit Rev Toxico, 37: 113-115.

Kerswill CJ, Edwards HE 1967. Fish losses after forest spraying with insecticides in New Brunswick, 19521962, as shown by caged specimens and other observations. J Fish Res Bd, 24: 709-729.

Krishnan K, Brodeur J 1994. Toxic interactions among environmental pollutants: corroborating laboratory observations with human experience. Environ Health Perspect, 102, 90: 11-17.

Lotti M, Johnson MK 1978. Neurotoxicity of organosphosphate pesticides: predictions can be based on in vitro studies with hen and human enzymes. Arch Toxicol, 41: 215-221.

Ludke JL, Hill EF, Dieter MP 1975. Cholinesterase (ChE) response and related mortality among birds fed ChE inhibitors. Arch Environ Contamin Toxicol, 3: 121.

Mansour SA, Heikal TM 2001. Xenobiotics interactions. A further study on the use of biochemical markers to analyze the joint action of insecticide mixtures in the rat. J. Egypt. Ger Soc Zool, 35 (E): 31-49. Mansour SA 2004. Pesticide exposure- Egyptian scene. Toxicology, 198: 91-115.

Peakall DB 1985. Behavioral responses of birds to pesticides and other contaminants. Residue Rev, 96: 45-77.

Pearce PA, Peakall DB, Erskine A J 1976. Impact on forest birds of the 1975 spruce budworm operation in New Brunswick. CWS Progress Note \# 61.

Rudolph SG, Zinkl JG, Anderson DW, Shea PJ 1984. Preycapturing ability of American Kestrels fed DDE and acephate and acephate alone. Arch Environ Contamin Toxicol, 13: 367-372.

Tonner M, Vavra V, Syrovatka O, Soldan T 1983. Einfluss der Luftbespritzung genen grauen Larchenwicker (Zeiraphera diniana) auf die Entomofauna des Rhithrons in Krkonose (Riesengebirge). Vest Cs Spol Zool, 47: 293-303.

Warner RE, Peterson KK, Borgman L 1966. Behavioural pathology in fish: a quantitative study of sub lethal pesticide toxication. J Appl Ecol, 3 (Suppl): 223247.

WHO 1995. Expert consultation on the application of risk analysis to food standards issues, 13-17 March 1995, Geneva: WHO.

Wilson LK, Harris M, Elliott JE 1999. Contaminants in wildlife indicator species from the Fraser Basin. In: $\mathrm{C}$ Gray, T Tuominen (Eds.): Health of the Fraser River Aquatic Ecosystem: A Synthesis of Research Conducted Under the Fraser River Action Plan. Environment Canada, Vancouver, BC. DOE FRAP 1998-11.

Wilson LK, Elliott JE, Szeto S, Vernon B 1996. Retention of insecticide in the granule and its potential hazard to wildlife. Presented at the 17th Annual SETAC (Society of Environmental Toxicology and Chemistry) Meeting, 17-21 November 1996, Washington, DC. Poster Abstract No. 526.

Wilson LK, Moul IE, Langelier KM, Elliott JE 1995. Summary of Bird Mortalities in British Columbia and the Yukon, 1963-1994. CWS Technical Report No. 249. Canadian Wildlife Service, Pacific and Yukon Region, P.205.

Yadav SK 2007. Soil Ecology. New Delhi: A P H Publishers. 